\title{
Protection of rat liver against hepatic ischemia-reperfusion injury by a novel selenocysteine-containing 7-mer peptide
}

\author{
QIANQIAN JIANG ${ }^{1,2}$, YU PAN $^{3}$, YUPENG CHENG $^{1}$, HUILING LI $^{1}$ and HUI $\mathrm{LI}^{2}$ \\ ${ }^{1}$ Pharmaceutical College, Heilongjiang University of Chinese Medicine, Harbin, Heilongjiang 150040; \\ ${ }^{2}$ Department of Biochemistry and Molecular Biology, Basic Medical Science College, Harbin Medical University, \\ Harbin, Heilongjiang 150081; ${ }^{3}$ School of Environment and Chemical Engineering, \\ Heilongjiang University of Science and Technology, Harbin, Heilongjiang 150022, P.R. China
}

Received July 21, 2015; Accepted June 13, 2016

DOI: $10.3892 / \mathrm{mmr} .2016 .5464$

\begin{abstract}
Hepatic ischemia-reperfusion (I-R) injury causes acute organ damage or dysfunction, and remains a problem for liver transplantation. In the I-R phase, the generation of reactive oxygen species aggravates the injury. In the current study, a novel selenocysteine-containing 7-mer peptide (H-Arg-Sec-Gly-Arg-Asn-Ala-Gln-OH) was constructed to imitate the active site of an antioxidant enzyme, glutathione peroxidase (GPX). The 7-mer peptide which has a lower molecular weight, and improved water-solubility, higher stability and improved cell membrane permeability compared with other GPX mimics. Its GPX activity reached $13 \mathrm{U} / \mu \mathrm{mol}$, which was 13 times that of ebselen (a representative GPX mimic). The effect of this GPX mimic on I-R injury of the liver was assessed in rats. The 7-mer peptide significantly inhibited the increase in serum hepatic amino-transferases, tissue malondialdehyde, nitric oxide contents, myeloperoxidase activity and decrease of GPX activity compared with I-R tissue. Following treatment with the 7-mer peptide, the expression of B-cell CLL/lymphoma-2 (Bcl-2) was significantly upregulated at the mRNA and protein level compared with the I-R group, as determined by reverse transcription-polymerase chain reaction and immunohistochemistry, respectively. By contrast, Bcl-2 associated $\mathrm{X}$ protein (Bax) was downregulated by the 7-mer peptide compared the I-R group. Histological and ultrastructural changes of the rat liver
\end{abstract}

Correspondence to: Professor Hui Li, Department of Biochemistry and Molecular Biology, Basic Medical Science College, Harbin Medical University, 194 Xuefu Road, Harbin, Heilongjiang 150081, P.R. China

E-mail: lihui@ems.hrbmu.edu.cn

Professor Yupeng Cheng, Pharmaceutical College, Heilongjiang University of Chinese Medicine, 24 Heping Road, Harbin, Heilongjiang 150040, P.R. China

E-mail: yupengcheng@msn.com

Key words: hepatic ischemia-reperfusion, 7-mer peptide, glutathione peroxidase, reactive oxygen species, selenocysteine tissue were also compared among the experimental groups. The results of the current study suggest that the 7-mer peptide protected the liver against hepatic I-R injury via suppression of oxygen-derived free radicals and regulation of Bcl-2 and Bax expression, which are involved in the apoptosis of liver cells. The findings of the present study will further the investigation of the 7-mer peptide as an effective therapeutic agent in hepatic I-R injury.

\section{Introduction}

A period of ischemia is required for a number of surgical procedures on the liver, including when dealing with hepatic trauma, resecting large intrahepatic lesions and transplanting. Ischemia causes tissue damage, however the liver is subjected to a further insult when restoring the blood supply, which is termed ischemia-reperfusion (I-R) injury (1). In the hepatic I-R period, several cellular function changes, including generation of reactive oxygen species (ROS), inflammatory cytokines and chemokines, may result in cell injury, triggering the apoptotic pathway and leading to organ damage or dysfunction (2). As numerous studies have reported (3-5) that generation of ROS and oxidant stress are the most vital mechanisms in I-R injury, the ROS scavengers should be considered as therapeutic agents for hepatic I-R. Under normal conditions, endogenous antioxidants, including vitamins $\mathrm{C}$ and $\mathrm{E}$, and the antioxidant enzymes superoxide dismutase (SOD), catalase and glutathione peroxidase (GPX) have the capacity to scavenge ROS products. However, in ischemic conditions, these defense mechanism fail to protect tissues from oxidative damage because of overproduction of oxygen radicals, inactivation of antioxidant enzymes and consumption of antioxidants in the ischemic tissue (6). SOD (7), allopurinol (8), and $\mathrm{N}$-acetylcysteine (9) have all been previously demonstrated to attenuate hepatic I-R injury.

GPX is a mammalian selenium-containing antioxidant enzyme, which scavenges peroxides and $\mathrm{H}_{2} \mathrm{O}_{2}$ using glutathione (GSH), and is associated with various ROS-mediated diseases (10). Due to its poor stability, limited availability and difficulty in preparation, various artificial GPX mimics have been made as free radical scavengers, including ebselen (11). Based on the structure of the active site in bovine GPX (12), the 
present study constructed a novel selenocysteine-containing 7-mer peptide (H-Arg-Sec-Gly-Arg-Asn-Ala-Gln-OH), with GPX activity that reaches $13 \mathrm{U} / \mu \mathrm{mol}$, which is 13 times that of ebselen. The 7-mer peptide is advantageous due to low molecular weight, improved cell membrane permeability, good water-solubility and stability compared with other previous GPX mimics. Encouraged by these advantages, the current study evaluated the effects of the 7-mer peptide on the liver damage induced by hepatic I-R injury using a rat model.

\section{Materials and methods}

Animals. All the animal experiments were performed using a protocol approved by the Animal Care and Research Committee of Heilongjiang University of Chinese Medicine (Harbin, China). Clean, healthy adult male Wistar rats, weighing 200-250 g, were obtained from the Laboratory Animal Center of Heilongjiang University of Chinese Medicine, provided with standard pellet food and tap water in individual cages with 12 -h light/dark cycle at $22+2^{\circ} \mathrm{C}$.

Animal administration and induction of liver I-R model. Rats $(n=48)$ were equally and randomly assigned into four experimental groups as follows: Sham operation group (control group, $n=12$ ), I-R group (physiological saline-treatment for $5 \mathrm{~min}$ prior to $\mathrm{I}-\mathrm{R}, \mathrm{n}=12)$, the 7 -mer $+\mathrm{I}-\mathrm{R}$ group $(15 \mu \mathrm{mol} / \mathrm{kg}$ 7 -mer peptide treatment for $5 \mathrm{~min}$ prior to $\mathrm{I}-\mathrm{R}, \mathrm{n}=12)$, the 7 -mer peptide control group $(15 \mu \mathrm{mol} / \mathrm{kg} 7$-mer peptide treatment without I-R, $\mathrm{n}=12$ ).

The model of partial hepatic I-R was performed using a published protocol (13). All rats were fasted for $12 \mathrm{~h}$ preceding the operation but provided with access to drinking water. The rats were anesthetized with an intraperitoneal injection of $40 \mathrm{mg} / \mathrm{kg}$ pentobarbital sodium (8\%; Sigma-Aldrich, St. Louis, $\mathrm{MO}, \mathrm{USA})$ and placed supinely on a heating pad $\left(36-37^{\circ} \mathrm{C}\right)$. At $5 \mathrm{~min}$ prior to occlusion, the 7 -mer peptide $(15 \mu \mathrm{mol} / \mathrm{kg}$ body weight) or physiological saline alone was intravenously injected into the tongue. A midline incision was made on the abdominal wall of the rats. The left portal vein and hepatic artery were occluded with a micro-clip for $60 \mathrm{~min}$, then the clip was removed to initiate hepatic reperfusion. The sham control group underwent the same protocol without vascular occlusion. Occlusion was verified visually by the change in liver color to a paler shade and upon reperfusion to a blush. Rats were sacrificed by cervical dislocation under anesthesia $(8 \%$ pentobarbital sodium) $2 \mathrm{~h}$ after reperfusion, blood samples and liver tissue (from the ischemic lobe) were taken for analysis.

Alanine aminotransferase (ALT; cat. no. C009-1), aspartate aminotransferase (ASTcat. no. C010-1), lactate dehydrogenase (LDH; cat. no. A020-1), myeloperoxidase (MPO; cat. no. A044), malondialdehyde (MDA cat. no. A003-1) and nitric oxide (NO; cat. no. A012) detection kits were purchased from Nanjing Jiancheng Bioengineering Institute (Nanjing, China). Rabbit anti-rat B-cell CLL/lymphoma-2 (Bcl-2; cat. no. ZA-0536) and Bcl-2 associated X protein (Bax; cat. no. ZA-0611) monoclonal antibodies were purchased from Zhongshan Jinqiao Biotechnology Co., Ltd. (Beijing, China). All other chemicals used were of analytical or reagent grade.
GPX-like activity assay of the 7-mer peptide. The 7-mer peptide (H-Arg-Sec-Gly-Arg-Asn-Ala-Gln-OH) was synthesized by GL Biochem, Ltd. (Shanghai, China). The catalytic activity was determined by the method of Wilson (14). The reaction was carried out at $37^{\circ} \mathrm{C}$ in $700 \mu \mathrm{l}$ reaction solution containing $50 \mathrm{mM}$ pH 7.0 sodium phosphate buffer, $1 \mathrm{mM}$ EDTA, $1 \mathrm{mM}$ sodium azide, $1 \mathrm{mM}$ GSH, $0.25 \mathrm{mM}$ nicotinamide adenine dinucleotide phosphate (NADPH), $1 \mathrm{U}$ of GSH reductase, $10-50 \mu \mathrm{M}$ of the mimic. The reaction was initiated by addition of $0.5 \mathrm{mM} \mathrm{H} \mathrm{O}_{2}$ or $\mathrm{CuOOH}$. The activity was determined by the decrease of NADPH absorption at $340 \mathrm{~nm}$ using a Lambda $750 \mathrm{UV} /$ Visable/Near Infrared spectrophotometer (PerkinElmer, Inc., Waltham, MA, USA). Background absorption was run without mimic and was subtracted. The activity unit was defined as the amount of the mimic that utilized $1 \mu \mathrm{mol}$ NADPH per min. The activity was expressed in $\mathrm{U} / \mu \mathrm{mol}$ of the GPX mimic.

Blood chemistry assay. Following the reperfusion period, $5 \mathrm{ml}$ blood was collected from the abdominal aorta. The blood sample was centrifuged at $1200 \mathrm{x}$ g for $10 \mathrm{~min}$ at room temperature to separate serum for analysis. Liver injury was assessed by measuring the levels of ALT (a specific marker of hepatic parenchymal injury), AST (a nonspecific marker of hepatic injury) and LDH (a marker of nonspecific cellular injury) in the serum. ALT, AST and LDH were assayed using assay kits (Nanjing Jiancheng Bioengineering Institute) according to the manufacturer's instructions with a DG 8 standard biochemistry automatic analyzer (Nanjing Huadong Electronics Group Medical Equipment Co., Ltd., Nanjing, China).

MPO, MDA, NO assay. Liver tissue $\left(\sim 1 \mathrm{~cm}^{3}\right)$ was obtained $2 \mathrm{~h}$ after reperfusion. The samples were homogenized in $10 \mathrm{ml}$ ice-cold physiological saline and centrifuged at $12,000 \mathrm{x} \mathrm{g}$ for $20 \mathrm{~min}$ at $4^{\circ} \mathrm{C}$ to separate the supernatant, then liver MPO activity, MDA content, protein content and serum NO content were measured using assay kits (Nanjing Jiancheng Bioengineering Institute) according to the manufacturer's instructions.

GPX activity assay. GPX activity was determined using the method of Wilson (14) using the supernatant of tissue luminal content. The enzymatic reaction contained the following components: NADPH, reduced GSH and GSH reductase, and was initiated by addition of $\mathrm{H}_{2} \mathrm{O}_{2}$. The change in absorbance at $340 \mathrm{~nm}$ was monitored by a Lambda 750 UV-Visible-Near-Infrared spectrophotometer (PerkinElmer, Inc.). Activity was recorded as U/g protein in liver tissue.

Immunohistochemical assay. For immunohistochemical analysis, tissue samples were obtained $2 \mathrm{~h}$ after reperfusion, fixed in $4 \%$ paraformaldehyde for $30 \mathrm{~min}$ at room temperature and embedded in paraffin. Paraffin-embedded tissue sections $(5 \mu \mathrm{m})$ were cut from each specimen and then immersed in xylene followed by rehydration in graded alcohol. The sections were washed with the phosphate-buffered saline (PBS) for $5 \mathrm{~min}$, fixed in cold acetone for $10 \mathrm{~min}$, washed with PBS $3 \times 3$ min and then incubated with $3 \% \mathrm{H}_{2} \mathrm{O}_{2}$ in distilled water for $5 \mathrm{~min}$ to quench any endogenous peroxidase activity 
and subsequently immersed in PBS for $5 \mathrm{~min}$. The tissue sections were then blocked with normal goat serum (Jackson ImmunoResearch Laboratories, Inc., West Grove, PA, USA) for $10 \mathrm{~min}$ and incubated at $37^{\circ} \mathrm{C}$ for $2 \mathrm{~h}$ with monoclonal rabbit anti-rat Bcl-2 and Bax antibodies (diluted 1:200) at a concentration of $5 \mu \mathrm{g} / \mathrm{ml}$, then washed with PBS 3 times for 3 min each time The expression of $\mathrm{Bcl}-2$ and Bax proteins were detected by the labeled streptavidin biotin (LSAB) method using an LSAB kit (Abcam, Cambridge, UK) consisting of a blocking reagent, biotinylated link antibody and peroxidase-labeled streptavidin reagents. The peroxidase binding sites were detected using 3', 3'-diaminobenzidine and images were acquired using an IX-81 microscope (Olympus Corporation, Tokyo, Japan).

Reverse transcription-polymerase chain reaction ( $R T-P C R)$ assay. The tissue samples taken from the rats were immediately frozen in liquid nitrogen and then stored at $-80^{\circ} \mathrm{C}$ until use. Total RNA was extracted from liver tissue by the acid guanidinium thiocyanate-phenol-chloroform method and concentration determined by UV spectrophotometer. RT was performed on RNA $(1 \mu \mathrm{g})$ and cDNA amplified using One-Step RT-PCR kit (Takara Biotechnology Co., Ltd., Dalian, China) with the following conditions: $45^{\circ} \mathrm{C}$ for $30 \mathrm{~min} ; 94^{\circ} \mathrm{C}$ for $5 \mathrm{~min} ; 40$ cycles at $94^{\circ} \mathrm{C}$ for $30 \mathrm{sec}, 55^{\circ} \mathrm{C}$ for $30 \mathrm{sec}, 72^{\circ} \mathrm{C}$ for $2 \mathrm{~min}$; and final step of $72^{\circ} \mathrm{C}$ for $10 \mathrm{~min}$. Primers used in the PCR reactions were as follows: $\mathrm{Bcl}-2$, sense 5'-CCCCTTCATCCAAGAATGC-3', antisense 5'-TTC CACAAAGGCATCCCAG-3', producing a 623 bp product; Bax, sense 5'-CCACCAGCTCTGAACAGTTCA-3', antisense 5'-TGAGGACTCCAGCCACAAAG-3', producing a 506 bp product. The PCR reaction products were separated by electrophoresis on $0.8 \%$ agarose gel and stained with ethidium bromide. Digital images were assessed with image analysis software and mRNA expressions were evaluated by the band intensity ratios of $\mathrm{Bcl}-2$ and Bax and presented as percentage of $\beta$-actin levels.

Histological analysis. Liver tissue samples were obtained following reperfusion and immediately fixed in $4 \%$ paraformaldehyde for $4 \mathrm{~h}$. The samples were subsequently transferred into $70 \%$ ethanol then embedded in paraffin, sectioned $(4 \mu \mathrm{m})$, and stained with hematoxylin (for $5 \mathrm{~min}$ ) and eosin (for $3 \mathrm{~min}$ ) for examination. The samples were imaged using an IX-81 microscope.

Electron microscopy analysis. Samples of small bowel tissue were obtained following reperfusion and immediately fixed in $2.5 \%$ glutaraldehyde at $4^{\circ} \mathrm{C}$ until they were subsequently fixed in $1 \%$ osmic acid. The fixed tissue was dehydrated in graded alcohol, embedded in Epon 812 epoxy resin, and cut into $4 \mu \mathrm{m}$-thin sections using the LKB super-thin sectioning machine. The sections were stained with acetate double oxygenic uranium (30 min) and citrate lead $(8 \mathrm{~min})$ for examination under a JEM-1200 EX transmissive electron microscope.

Statistical analysis. All data are expressed as the mean \pm standard deviation. Statistical analysis was performed using one-way analysis of variance and post-hoc
Student-Newman-Keuls test for multi-group comparisons with SPSS software (version 19.0; IBM SPSS, Armonk, NY, USA). $\mathrm{P}<0.05$ was considered to indicate a statistically significant difference.

\section{Results}

Characterization of the designed 7-mer peptide. The key for GPX imitation is to generate high affinity for GSH. Based on the main amino residues in active site of the bovine GPX, the sequence of the 7-mer peptide was designed as follows: $\mathrm{H}$-Arg-Sec-Gly-Arg-Asn-OH; this sequence increases Gly flexibility, Sec is the catalytic site of the 7-mer peptide, Arg and Asn form salt bridges and a hydrogen bond with the GSH molecule. Glutamine (Glu) is the most abundant amino acid in the plasma and is important for modulating inflammatory responses, oxidative stress and apoptosis (15). It has previously been reported that Glu protected the gut, heart and skeletal muscle against I-R injury by preserving the GSH content in the tissues (16). However, the limited solubility and poor stability hampered the application of Glu as a therapeutic for I-R injury. Aiming to solve this problem, alanine (Ala) was employed to enhance the stability and solubility of Glu in the form of alanyl-Glu dipeptide, which had previously been demonstrated to protect rats from hepatic I-R injury (17). Thus, in the current study, Ala-Gln was added to the C-terminal of the peptide in order to improve the activity and stability of the 7-mer peptide.

GPX-like activity of the 7-mer peptide. The activity of the 7-mer peptide-catalyzed reduction of $\mathrm{H}_{2} \mathrm{O}_{2}$ by $\mathrm{GSH}$ is presented in Table I. The GPX activity of the 7-mer peptide was $13 \mathrm{U} / \mu \mathrm{mol}$, which is $\sim 13$ times that of ebselen $(0.99 \mathrm{U} / \mu \mathrm{mol})$, a well-known GPX mimic.

Effect of the 7-mer peptide on liver injury. Liver injury was assessed by measuring serum levels of ALT, AST and LDH. Compared with sham-operated rats, the ALT (Fig. 1; $\mathrm{P}<0.01$ ), AST (Fig. 2; P<0.01), LDH (Fig. 3; P<0.01) levels in serum were significantly increased in I-R liver, which demonstrated the development of hepatocellular injury. Whereas, for the I-R rats pretreated with the 7-mer peptide, no significant increase in ALT, AST and LDH levels were detected. In the 7-mer peptide only group, no significant effect on ALT, AST and LDH serum levels was detected, indicating that the 7-mer peptide exerts no I-R injury to the rats.

Effect of the 7-mer peptide on hepatic neutrophil recruitment. MPO activities in liver tissues were analyzed as the index of hepatic neutrophil recruitment. A unit of MPO activity is defined as that which degrades $1 \mu \mathrm{mol} \mathrm{H}_{2} \mathrm{O}_{2} / \mathrm{min}$ at $25^{\circ} \mathrm{C}$. In the I-R group, I-R caused a significantly increased the MPO activity in the liver compared with sham control $(\mathrm{P}<0.01)$. This increase of MPO activity following reperfusion in the I-R group was significantly inhibited by pretreatment of the liver tissues with the 7-mer peptide ( $\mathrm{P}<0.01$; Fig. 4).

Inhibition of lipid peroxidation by the 7-mer peptide. MDA is the final product of lipid peroxidation, liver tissues were assayed for MDA content as a marker of hepatic oxidative 
stress. Hepatic I-R injury significantly increased the MDA content in the liver compared with the sham control group $(\mathrm{P}<0.01)$. The I-R-induced increase in MDA level was significantly prevented by the 7 -mer peptide treatment $(\mathrm{P}<0.01$; Fig. 5).

Reduction of NO content by the 7-mer peptide. NO is a potent vasodilator that reacts with superoxide to form the oxidant peroxynitrite, which is considered to be a strong cytotoxic agent. In the current study, NO content was significantly increased in the hepatic I-R injury group compared with the sham control group $(\mathrm{P}<0.01)$, and the 7 -mer peptide treatment inhibited the increase in NO level caused by I-R $(\mathrm{P}<0.01$; Fig. 6).

Effect of the 7-mer peptide on liver GPX activity. GPX, an endogenous antioxidant enzyme, usually limits damage caused by oxygen derived free radicals, however its level falls in response to increased free radicals. Following the $2-\mathrm{h}$ reperfusion period in the present study a significant decrease in GPX activity was observed in the I-R group compared with the sham control group $(\mathrm{P}<0.01)$, whereas the decrease of GPX activity in the I-R group was inhibited by the 7-mer peptide treatment $(\mathrm{P}<0.01$; Fig. 7$)$.

Inhibition of the expression levels of $\mathrm{Bcl}-2$ and Bax by the 7-mer peptide. The protein expression levels of $\mathrm{Bcl}-2$ and $\mathrm{Bax}$ protein in the liver following I-R were evaluated by immunohistochemical analysis. In the I-R group, there was weak expression of $\mathrm{Bcl}-2$ protein (Fig. 8). By contrast, strong immunoreactivity for Bax expression was observed in the I-R group $2 \mathrm{~h}$ after reperfusion compared with the sham control group (Fig. 9). In the 7-mer peptide + I-R group, the expression of $\mathrm{Bcl}-2$ was significantly upregulated compared with the I-R group $(\mathrm{P}<0.01)$, and Bax overexpression was significantly suppressed $(\mathrm{P}<0.01$; Fig. 10).

Inhibition of hepatic mRNA expression levels of Bcl-2 and Bax by the 7-mer peptide. To determine the mRNA expression levels of the apoptosis regulatory genes, mRNA transcript levels for Bcl-2 and Bax were assessed by RT-PCR. The band intensity ratios of Bcl-2 and Bax normalized to $\beta$-actin were compared among the sham, I-R and the 7-mer peptide-treated groups. As demonstrated in Fig. 11, hepatic I-R significantly increased the mRNA expression of Bax compared with sham control $(\mathrm{P}<0.01)$, with the expression of Bcl-2 decreased $(\mathrm{P}<0.05)$. Administration of the 7-mer peptide significantly enhanced the Bcl-2 mRNA expression compared with the I-R group $(\mathrm{P}<0.01)$ and suppressed hepatic I-R-induced mRNA overexpression of Bax $(\mathrm{P}<0.01$; Fig. 11).

Histological changes. Following 2-h reperfusion, severe swelling induced by hepatic I-R injury was observed along with abundant fatty and vacuolation degeneration in hepatocytes compared with the sham control group. The hepatic sinus compartment became narrow or disappeared. Hemorrhage and even derangement of cell constitution were also observed. In the 7-mer peptide-treated I-R group, these changes were reduced and the hepatic cellular structure remained clear (Fig. 12).

Ultrastructural changes of organelles in cytoplasm. The ultrastructural changes to the organelles in the cytoplasm
Table I. GPX activities of the 7-mer peptide and other GPX mimics.

\begin{tabular}{lcc}
\hline Species & Substrate & Activity $(\mathrm{U} / \mu \mathrm{mol})$ \\
\hline 7-mer peptide & $\mathrm{H}_{2} \mathrm{O}_{2}$ & 13.0 \\
Ebselen & $\mathrm{H}_{2} \mathrm{O}_{2}$ & 0.99 \\
Seleno-cyclodextrin & $\mathrm{H}_{2} \mathrm{O}_{2}$ & 7.4 \\
Native GPX & $\mathrm{H}_{2} \mathrm{O}_{2}$ & 5780 \\
\hline
\end{tabular}

GPX, glutathione peroxidase.

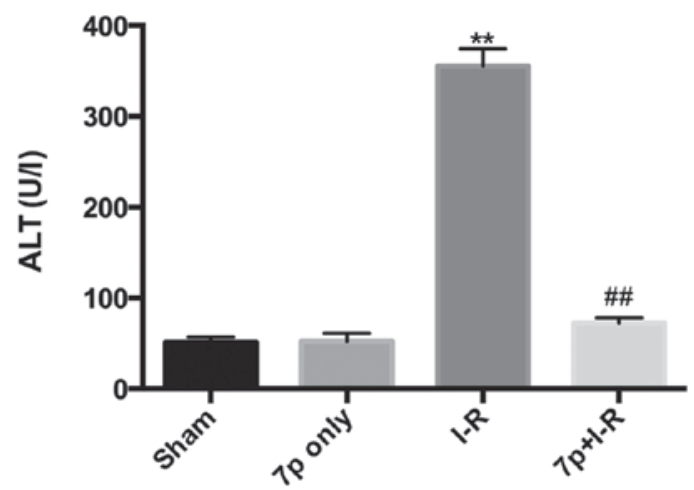

Figure 1. Effect of the 7p on ALT activity in serum following hepatic reperfusion. Student's $t$ test was used, values are mean \pm standard deviation of three independent experiments. ${ }^{* *} \mathrm{P}<0.01$ vs. sham group; ${ }^{\# \#} \mathrm{P}<0.01$ vs. I-R injury group. ALT, alanine aminotransferase; 7p, 7-mer peptide; I-R, ischemia-reperfusion.

were observed by electron microscopy among the experimental groups. As demonstrated in Fig. 13, in the I-R injury group, the rough endoplasmic reticulum was dilated in the majority of cells. Additionally, the clearance of dying cells was increased and the mitochondria in the hepatic cytoplasm were severely swollen. The nuclei were irregular in the I-R injury group, the nucleoli were visible and agglomeration of heterochromatin was also observed. The structure of organelles was not clear at $2 \mathrm{~h}$ after reperfusion compared with the sham and 7-mer peptide only groups. In the 7-mer peptide-treated I-R group, all of the described changes were observably reduced (Fig. 13).

\section{Discussion}

Liver ischemia during arterial occlusion, shock or organ transplantation is a common cause of hepatocyte death and liver failure. The ischemic cells manifest distinct biochemical, structural and functional alterations that finally lead to injury (18). The are numerous mediators involved in the pathogenesis of hepatic I-R injury. Among them, ROS and cell apoptosis are central to this process $(19,20)$.

Previous evidence has supported that ROS are produced at the moment of reperfusion initiation following hepatic ischemia, and free radicals are involved in the mechanism of hepatic graft failure during reperfusion in rats (21). Additionally, oxidative stress is also demonstrated to be sustained for a long time following clinical liver transplantation (22). Thus, 


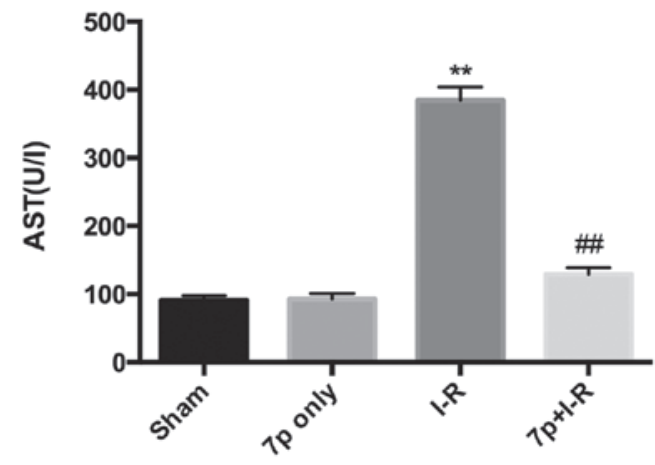

Figure 2. Effect of the 7p on AST activity in serum following hepatic reperfusion. Student's $t$ test was used, values are mean \pm standard deviation of three independent experiments. ${ }^{* *} \mathrm{P}<0.01$ vs. sham group; ${ }^{\# \#} \mathrm{P}<0.01$ vs. I-R injury group. AST, aspartate aminotransferase; 7p, 7-mer peptide; I-R, ischemia-reperfusion.

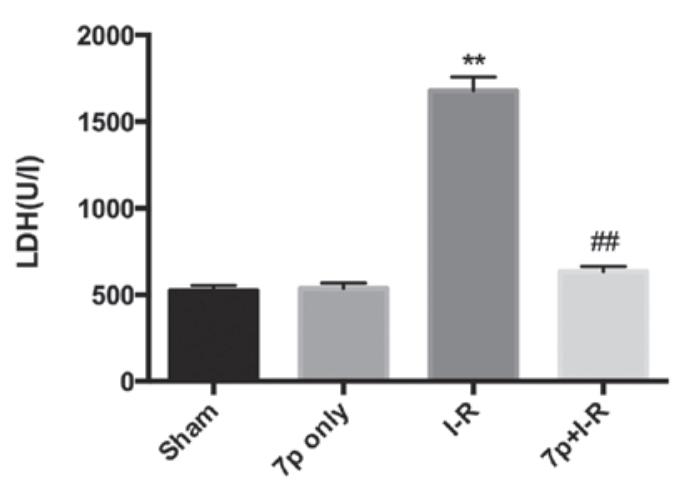

Figure 3. Effect of the 7p on LDH activity in serum following hepatic reperfusion. Student's $t$ test was used, values are mean \pm standard deviation of three independent experiments. ${ }^{* *} \mathrm{P}<0.01$ vs. sham group; ${ }^{\# \#} \mathrm{P}<0.01$ vs. I-R injury group. LDH, lactate dehydrogenase; 7p, 7-mer peptide; I-R, ischemia-reperfusion.

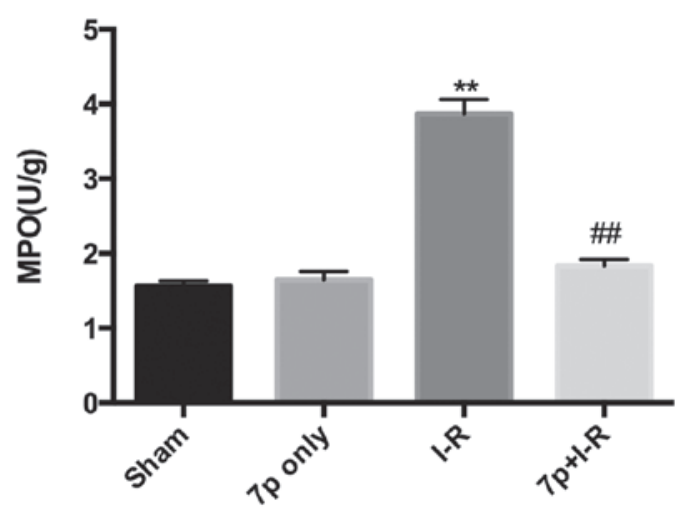

Figure 4. Effect of the 7p on liver MPO activity following hepatic reperfusion. Student's $t$ test was used, values are mean \pm standard deviation of three independent experiments. ${ }^{* *} \mathrm{P}<0.01$ vs. control group; ${ }^{\# \#} \mathrm{P}<0.01$ vs. I-R injury group. MPO, myeloperoxidase; 7p, 7-mer peptide; I-R, ischemia-reperfusion.

reduction of hepatic oxidative stress, including via pretreatment with ROS-scavengers, may effectively protect liver against I-R injury and transplant failure.

A novel selenocysteine-containing 7-mer peptide was constructed in the present study to imitate GPX, an

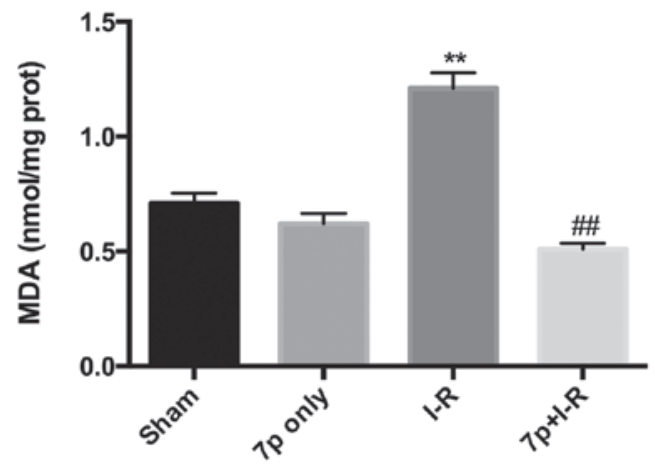

Figure 5. Effect of the 7p on liver MDA content following hepatic reperfusion. Student's $t$ test was used, values are mean \pm standard deviation of three independent experiments. ${ }^{* *} \mathrm{P}<0.01$ vs. sham group; ${ }^{\# \#} \mathrm{P}<0.01$ vs. I-R injury group. MDA, malondialdehyde; 7p, 7-mer peptide; I-R, ischemia-reperfusion.

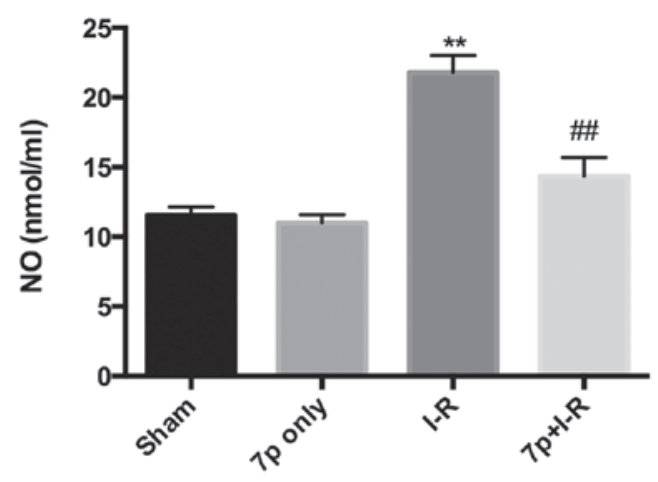

Figure 6. Effect of the 7p on liver NO content following hepatic reperfusion. Student's $t$ test was used, values are mean \pm standard deviation of three independent experiments. ${ }^{* *} \mathrm{P}<0.01$ vs. sham group; ${ }^{\#} \mathrm{P}<0.05$ vs. I-R injury group. $\mathrm{NO}$, nitric oxide; 7p, 7-mer peptide; I-R, ischemia-reperfusion.

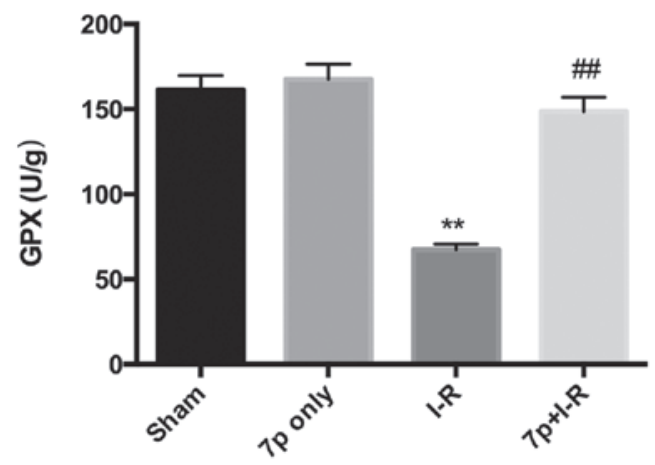

Figure 7. Effect of the 7p on liver GPX activity following hepatic reperfusion. Student's $t$ test was used, values are mean \pm standard deviation of three independent experiments. ${ }^{* *} \mathrm{P}<0.01$ vs. sham group; ${ }^{\# \#} \mathrm{P}<0.01$ vs. I-R injury group. GPX, glutathione peroxidase; 7p, 7-mer peptide; I-R, ischemia-reperfusion.

important antioxidant enzyme, which is an oxygen radical scavenger and has the capacity to protect cells against oxidative damage. This study aimed to investigate whether the 7-mer peptide may exert a protective effect and by consequence be a potential pharmacological agent for hepatic I-R injury, which has previously been characterized as a complex process encompassing a number of mechanisms, including oxidant stress and apoptosis. The results of the present 
A

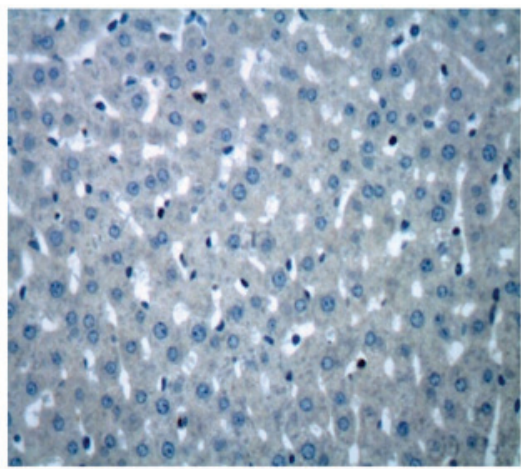

C

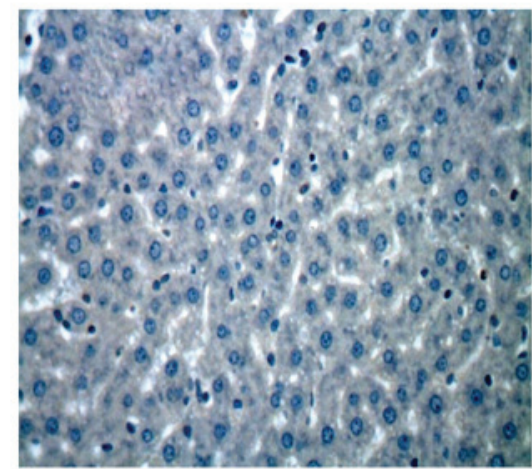

B

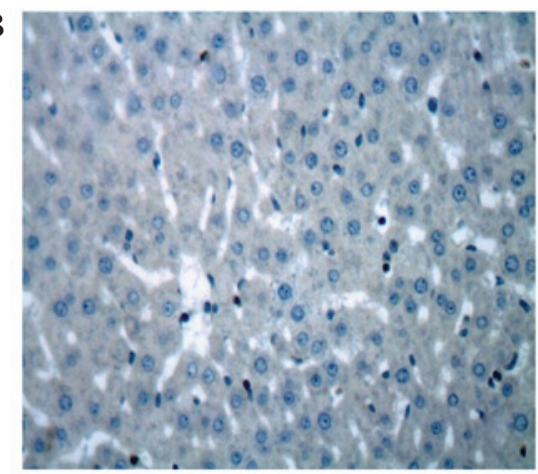

D

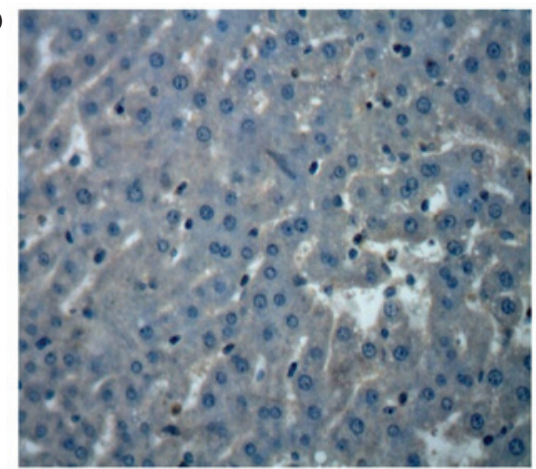

Figure 8. Immunohistochemical assay of B-cell CLL/lymphoma-2 in liver tissue. (A) Sham control group; (B) 7p control group; (C) I-R group; (D) 7p treated I-R group. Original magnification, $\mathrm{x} 400.7 \mathrm{p}, 7$-mer peptide; I-R, ischemia-reperfusion.

A

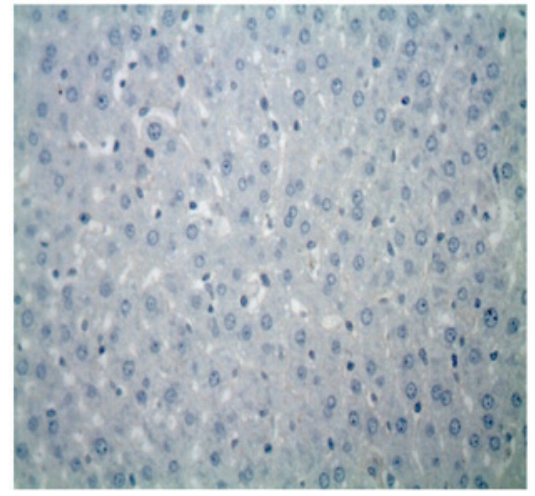

C

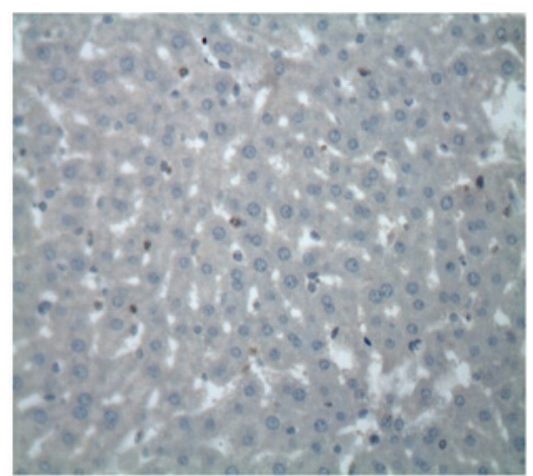

B

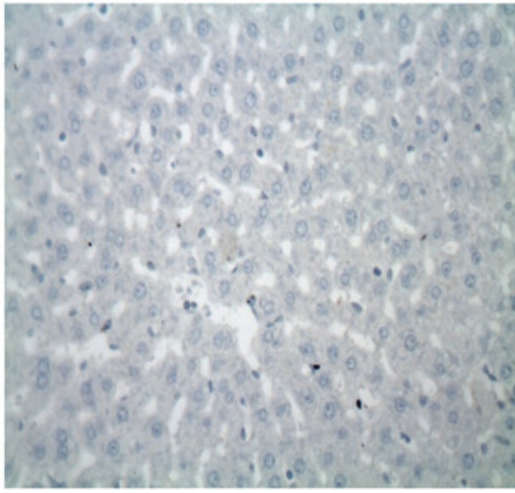

D

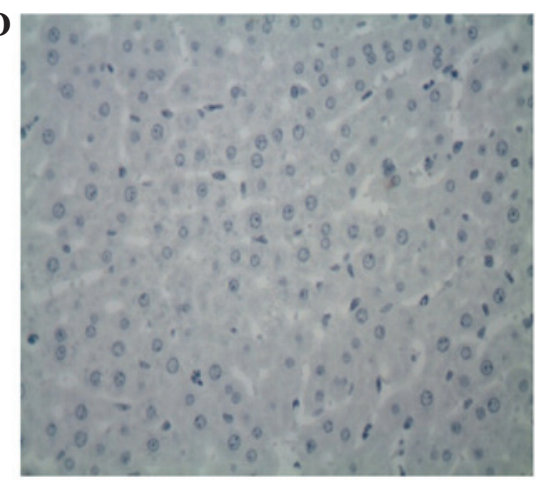

Figure 9. Immunohistochemical assay of Bcl-2 associated X protein in liver tissue. (A) Sham control group; (B) 7p control group; (C) I-R group; (D) 7p treated I-R group. Original magnification: $\mathrm{x} 400.7 \mathrm{p}, 7$-mer peptide; I-R, ischemia-reperfusion.

study indicated that the administration of the 7-mer peptide attenuated rat liver injury induced by I-R, demonstrated by the reduction of serum ALT and AST, hepatic MPO activity,
MDA content, NO content, attenuation of histopathological alterations, inhibition of GPX activity and decreased cell apoptosis. The results demonstrated that the 7-mer peptide 


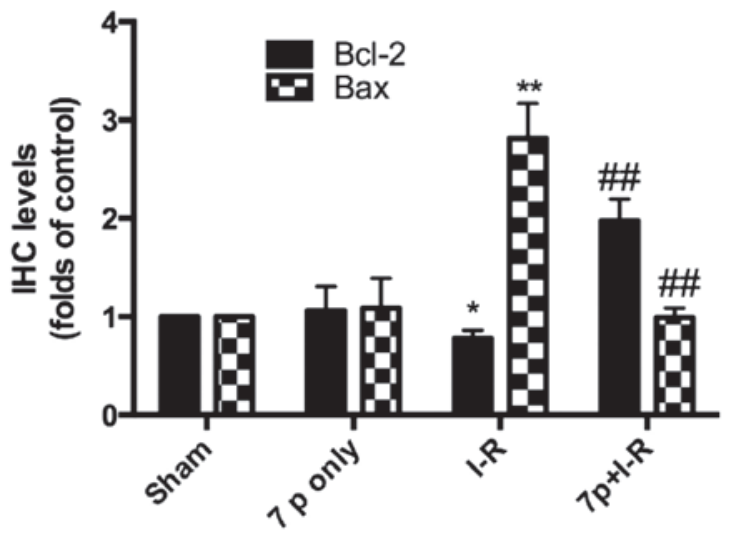

Figure 10. The IHC levels of Bcl-2 and Bax expression in liver tissue. Student's $t$ test was used, values are mean \pm standard deviation of three independent experiments. ${ }^{*} \mathrm{P}<0.05,{ }^{* *} \mathrm{P}<0.01$ vs. sham group; ${ }^{\# \#} \mathrm{P}<0.01$ vs. I-R injury group. IHC, immunohistochemistry; Bcl-2, B-cell CLL/lymphoma-2; Bax, Bcl-2 associated $\mathrm{X}$ protein; 7p, 7-mer peptide; I-R, ischemia-reperfusion.

exhibits various I-R-protective effects via antioxidation and antiapoptosis.

MPO is an enzyme expressed in leukocytes. Tissue MPO levels may indicate leukocyte infiltration into liver tissue following I-R (23). According to the findings of the present study, the 7-mer peptide inhibited the elevation of MPO activity following reperfusion and consequently protected against hepatic injury (Fig. 4). Additionally, lipid peroxidation was monitored by measuring MDA level, which represent from free radical damage to membrane components of the cells (24). Treatment with the 7-mer peptide significantly attenuated the increase of MDA concentration in the tissue (Fig. 5), the effect may be due to its capacity to eliminate ROS. The increase of superoxide and NO contents has been previously demonstrated lead to peroxynitrite-induced injury, and the increased NO production that occurs during reperfusion by an increase in inducible NO synthase activity promotes lipid peroxidation and cell damage (25). The current study indicated the marked elevation in NO level in the hepatic I-R injury was significantly attenuated by the 7-mer peptide (Fig. 6). This effect on NO generation in the liver tissue of I-R group supported that generation of NO may be caused by free radicals under oxidative stress. The 7-mer peptide, as a novel GPX mimic, protected the liver from the depletion of GPX activity (Fig. 7).

Oxygen-radical-induced apoptosis has also been previously reported to be involved in I-R injury and regarded as a central mechanism of injury during hepatic I-R. A number of genes regulate the apoptotic process. The family of $\mathrm{Bcl}-2$-associated proteins are involved in the regulation of apoptosis. Bcl-2, an anti-apoptotic protein, is known to prevent increased mitochondrial permeability, release of cytochrome $c$ and various caspases. As a member of the Bcl-2-associated protein family, Bcl-2 promotes cell survival through interactions with other Bcl-2 protein family members (26). Previous studies have indicated that over-expression of Bcl-2 protein reduced hepatocellular apoptosis following reperfusion and protected against hepatic I-R injury $(27,28)$. Bax, another member of the Bcl-2-associated protein family, forms homodimers to accelerate cell death or heterodimers with Bcl-2 to inhibit cell death. Changes in the ratio of Bcl-2 and
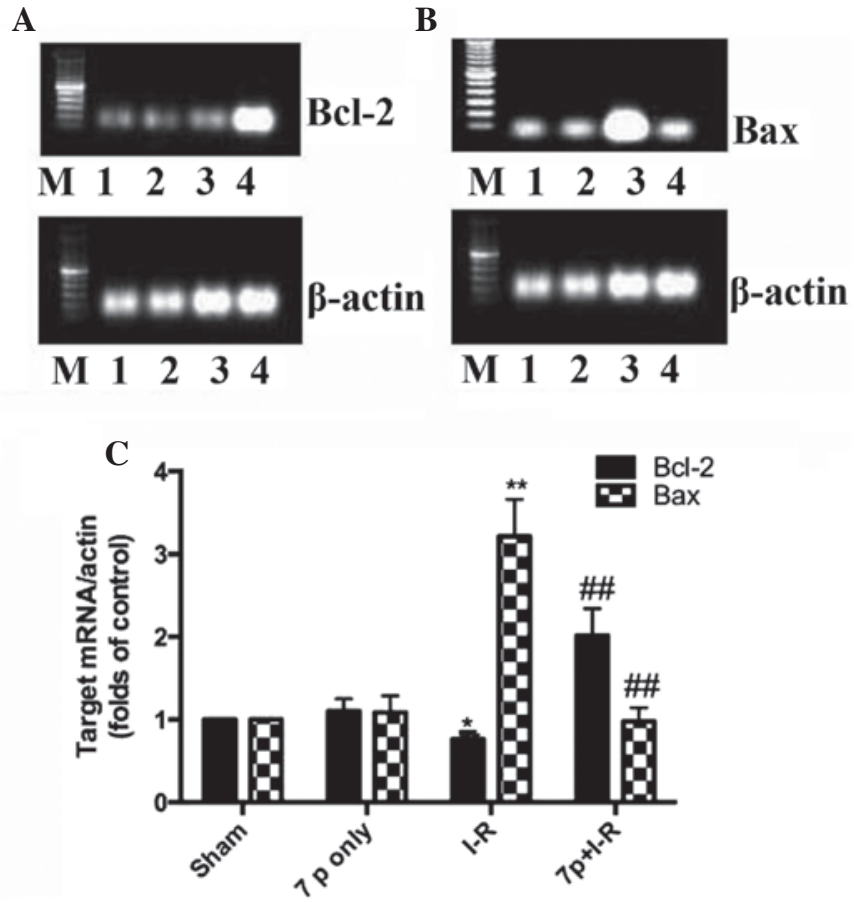

Figure 11. Semi-quantitative reverse transcription-polymerase chain reaction analysis of (A) Bcl-2 and (B) Bax mRNA expression in the liver tissue. $\beta$-actin was used as control. M: marker; Lane 1: sham control group; Lane 2: 7p control group; Lane 3: I-R group; Lane 4: 7p-treated I-R group. (C) Relative expression of Bcl-2 and Bax mRNA (\% $\beta$-actin) in the four groups were compared. Student's $t$ test was used, ${ }^{*} \mathrm{P}<0.05,{ }^{* *} \mathrm{P}<0.01$ vs. control group; ${ }^{\# \#} \mathrm{P}<0.01$ vs. I-R injury group. Bcl-2, B-cell CLL/lymphoma-2; Bax, Bcl-2 associated $\mathrm{X}$ protein; 7p, 7-mer peptide; I-R, ischemia-reperfusion.

Bax expression determine cell survival or death following apoptotic stimuli (29-31).

The present study examined cell apoptosis via RT-PCR and immunohistochemistry. According to the results, Bcl-2 was not observed to be overexpressed in the I-R group compared with the control, however the Bcl-2 mRNA and protein expression levels were increased in the 7-mer peptide-treated I-R group. By contrast, overexpression of Bax in rat liver was observed $2 \mathrm{~h}$ after reperfusion in the I-R group, and the 7-mer peptide pretreatment returned Bax to normal levels, indicating that the 7-mer peptide protected against hepatic I-R injury by upregulation of Bcl-2 and downregulation of Bax to inhibit I-R-induced apoptosis.

In summary, the present study constructed a novel GPX mimic and demonstrated the protective effect of the 7-mer peptide on hepatic I-R injury. Its protective mechanisms may be attributed to its free radical scavenging capacity and antioxidative activity by reducing the production of ROS and increasing the activity of GPX. The 7-mer peptide also exhibited antiapoptotic activity by regulating the expression of apoptotic genes associated with hepatic I-R. These results suggest that the 7-mer peptide could potentially be used for protecting the liver against hepatic I-R injury. The 7-mer peptide may be a potent antioxidant for use in pharmacological investigation.

\section{Acknowledgements}

This study was supported by the National Natural Science Foundation of China (grant no. 81573539), the 
A

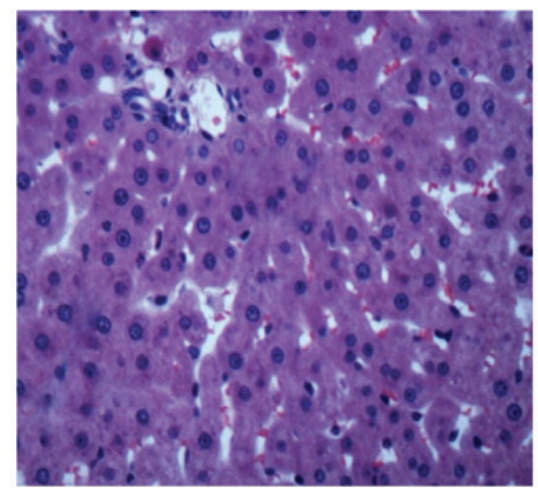

C

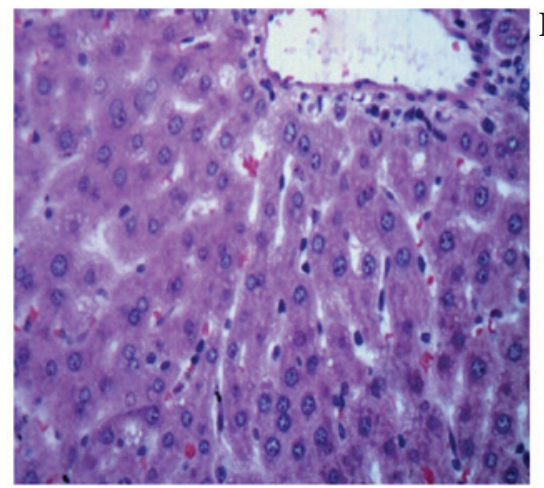

B

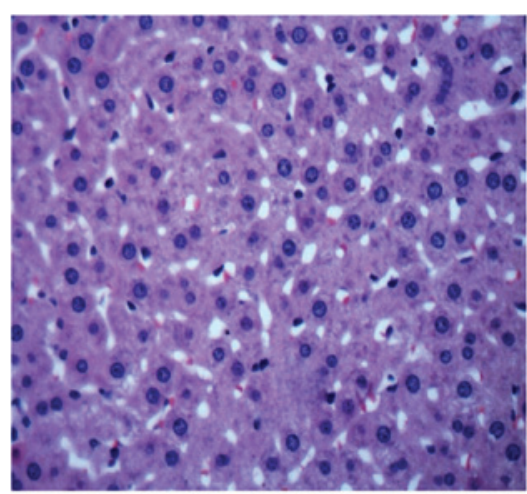

D

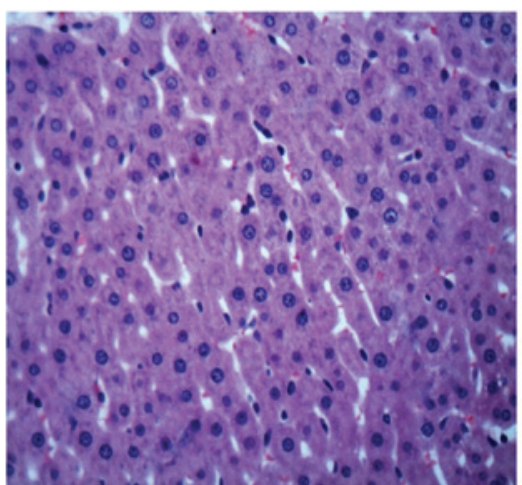

Figure 12. Microscopic histological changes of liver tissue following hematoxylin and eosin staining. (A) Sham control group; (B) 7p control group; (C) I-R group; (D) 7p treated I-R group. Original magnification, $x 400.7 p$, 7-mer peptide; I-R, ischemia-reperfusion.
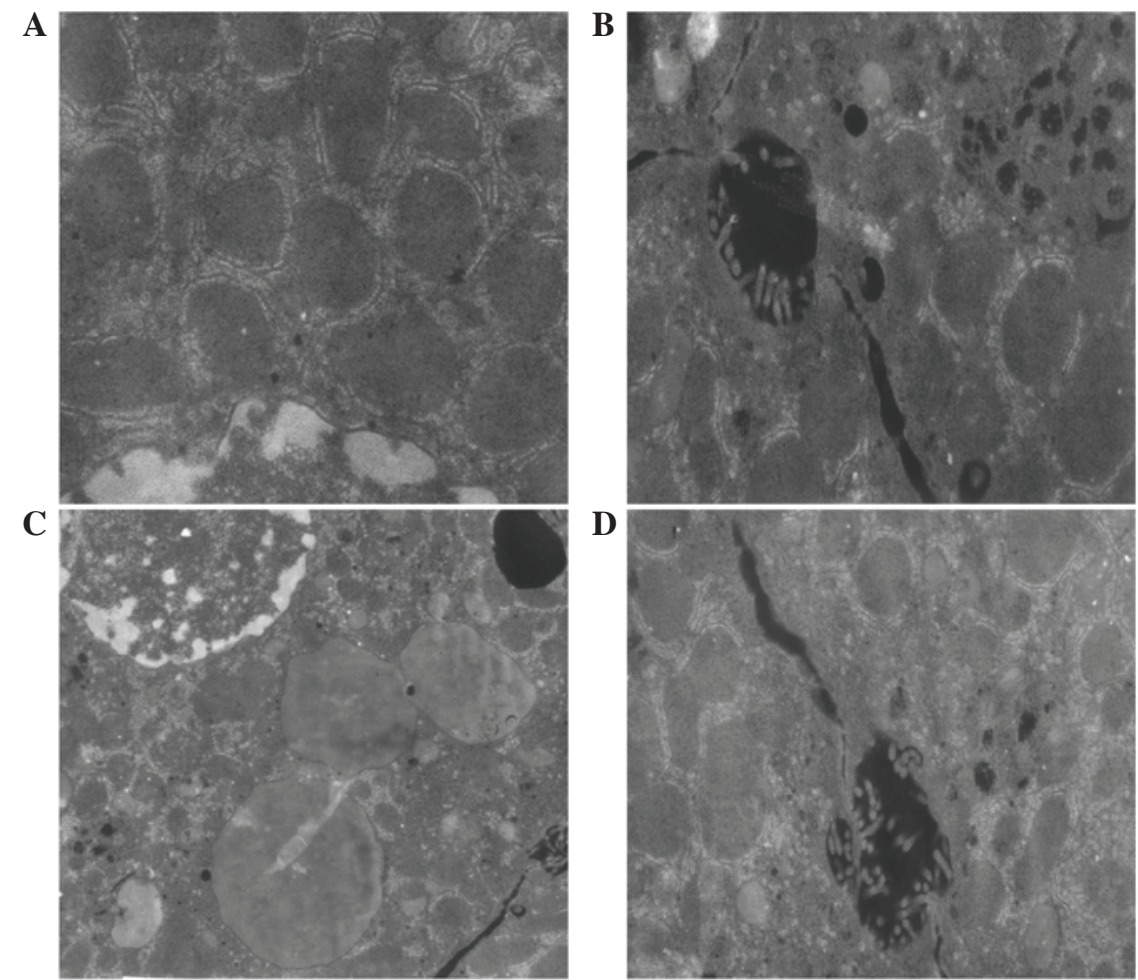

Figure 13. Hepatic ultrastructure changes following reperfusion under electron microscopy. (A) Sham control group; (B) 7p control group; (C) I-R group; (D) 7p treated I-R group. Original magnification, x12 K. 7p, 7-mer peptide; I-R, ischemia-reperfusion.

Natural Science Foundation of Heilongjiang Province (grant no. H2015042), the Excellent Creative Talents Support Project of Heilongjiang University of Chinese
Medicine (grant no. 2012RCQ12) and the Research Foundation of Heilongjiang University of Chinese Medicine (grant no. 201004). 


\section{References}

1. Eltzschig HK and Eckle T: Ischemia and reperfusion-from mechanism to translation. Nat Med 17: 1391-1401, 2011.

2. Klune JR and Tsung A: Molecular biology of liver ischemia/reperfusion injury: Established mechanisms and recent advancements. Surg Clin North Am 90: 665-677, 2010.

3. Taki-Eldin A, Zhou L, Xie HY, Chen KJ, Yu D, He Y and Zheng SS: Triiodothyronine attenuates hepatic ischemia/reperfusion injury in a partial hepatectomy model through inhibition of proinflammatory cytokines, transcription factors, and adhesion molecules. J Surg Res 178: 646-656, 2012.

4. Katsumi H, Nishikawa M, Yamashita $F$ and Hashida $M$ Prevention of hepatic ischemia/reperfusion injury by prolonged delivery of nitric oxide to the circulating blood in mice. Transplantation 85: 264-269, 2008.

5. Katsumi H, Nishikawa M, Yasui $H$, Yamashita $F$ and Hashida $M$ : Prevention of ischemia/reperfusion injury by hepatic targeting of nitric oxide in mice. J Control Release 140: 12-17, 2009.

6. Chan PH: Role of oxidants in ischemic brain damage. Stroke 27: 1124-1129, 1996.

7. Mizoe A, Kondo S, Azuma T, Fujioka H, Tanaka K, Hashida M and Kanematsu T: Preventive effects of superoxide dismutase derivatives modified with monosaccharides on reperfusion injury in rat liver transplantation. J Surg Res 73: 160-165, 1997.

8. Kusumoto K, Morimoto T, Minor T, Uchino J and Isselhard W. Allopurinol effects in rat liver transplantation on recovery of energy metabolism and free radical-induced damage. Eur Surg Res 27: 285-291, 1995.

9. Koeppel TA, Lehmann TG, Thies JC, Gehrcke R, Gebhard MM, Herfarth C, Otto G and Post S: Impact of N-acetylcysteine on the hepatic microcirculation after orthotopic liver transplantation. Transplantation 61: 1397-1402, 1996.

10. Lubos E, Loscalzo J and Handy DE: Glutathione peroxidase-1 in health and disease: From molecular mechanisms to therapeutic opportunities. Antioxid Redox Signal 15: 1957-1997, 2011.

11. Sies H: Ebselen, a selenoorganic compound as glutathione peroxidase mimic. Free Radic Bio Med 14: 313-323, 1993.

12. Epp O, Landensteine R and Wendel A: The refined structure of the selenoenzyme glutathione peroxidase at $0.2-\mathrm{nm}$ resolution. Eur J Biochem 133: 51-69, 1983.

13. Kohmoto J, Nakao A, Stolz DB, Kaizu T, Tsung A, Ikeda A, Shimizu H, Takahashi T, Tomiyama K, Sugimoto R, et al: Carbon monoxide protects rat lung transplants from ischemiareperfusion injury via a mechanism involving p38 MAPK pathway. Am J Transplant 7: 2279-2290, 2007.

14. Gavish M, Zakut R, Wilckek M and Givol D: Preparation of a semisynthetic antibody. Biochemistry 17: 1345-1351, 1978.

15. Santos AA, Braga-Neto MB, Oliveira MR, Freire RS, Barros EB, Santiago TM, Rebelo LM, Mermelstein C, Warren CA, Guerrant RL and Brito GA: Glutamine and alanyl-glutamine increase RhoA expression and reduce Clostridium difficile toxin-a-induced intestinal epithelial cell damage. Biomed Res Int 2013: 152052, 2013.

16. Jia CJ, Dai CL, Zhang X, Cui K, Xu F and Xu YQ: Alanylglutamine dipeptide inhibits hepatic ischemia-reperfusion injury in rats. World J Gastroenterol 12: 1373-1378, 2006.
17. Braga-Neto MB, Oliveira BM, Rodrigues RS, Noronha FJ, Leitao RF, Brito GA, Lima AA, Guerrant RL and Warren CA Protective effects of alanyl-glutamine supplementation against nelfinavir-induced epithelial impairment in IEC-6 cells and in mouse intestinal mucosa. Cancer Biol Ther 13: 1482-1490, 2012.

18. Dobashi K, Ghosh B, Orak JK, Singh I and Singh AK: Kidney ischemia-reperfusion: Modulation of antioxidant defenses. Mol Cell Biochem 205: 1-11, 2000.

19. KimHJ, Joe Y,Kong JS, Jeong SO, Cho GJ, Ryter SW and Chung HT: Carbon monoxide protects against hepatic ischemia/reperfusion injury via ROS-dependent Akt signaling and inhibition of glycogen synthase kinase 3B. Oxid Med Cell Longev 2013: 306421, 2013.

20. Pan S, Liu L, Pan H, Ma Y, Wang D, Kang K, Wang J, Sun B, Sun $\mathrm{X}$ and Jiang H: Protective effects of hydroxytyrosol on liver ischemia/reperfusion injury in mice. Mol Nutr Food Res 57: 1218-1227, 2013.

21. Connor HD, Gao W, Nukina S, Lemasters JJ, Mason RP and Thurman RG: Evidence that free radicals are involved in graft failure following orthotopic liver transplantation in the rat-an electron paramagnetic resonance spin trapping study. Transplantation 54: 199-204, 1992.

22. Burke A, FitzGerald GA and Lucey MR: A prospective analysis of oxidative stress and liver transplantation. Transplantation 74: 217-221, 2002

23. Wang XL, Liu HR, Tao L, Liang F, Yan L, Zhao RR, Lopez BL, Christopher TA and Ma XL: Role of iNOS-derived reactive nitrogen species and resultant nitrative stress in leukocytes-induced cardiomyocyte apoptosis after myocardial ischemia/reperfusion. Apoptosis 12: 1209-1217, 2007.

24. de Vries DK, Kortekaas KA, Tsikas D, Wijermars LG, van Noorden CJ, Suchy MT, Cobbaert CM, Klautz RJ, Schaapherder AF and Lindeman JH: Oxidative damage in clinical ischemia/reperfusion injury: A reappraisal. Antioxid Redox Signal 19: 535-545, 2013.

25. Simonsen U, Rodriguez-Rodriguez R, Dalsgaard T, Buus NH and Stankevicius E: Novel approaches to improving endothelium-dependent nitric oxide-mediated vasodilatation. Pharmacol Rep 61: 105-115, 2009.

26. Sheng M, Zhou Y, Yu W, Weng Y, Xu R and Du H: Protective effect of Berberine pretreatment in hepatic ischemia/reperfusion injury of rat. Transplant Proc 47: 275-282, 2015.

27. Wang C, Chen K, Xia Y, Dai W, Wang F, Shen M, Cheng P, Wang J, Lu J, Zhang Y, et al: N-acetylcysteine attenuates ischemia-reperfusion-induced apoptosis and autophagy in mouse liver via regulation of the $\mathrm{ROS} / \mathrm{JNK} / \mathrm{Bcl}-2$ pathway. PLoS One 9: e108855, 2014.

28. Zhao J, Ming Y, Wan Q, Ye S, Xie S, Zhu Y, Wang Y, Zhong Z, Li L and Ye Q: Gypenoside attenuates hepatic ischemia/reperfusion injury in mice via anti-oxidative and anti-apoptotic bioactivities. Exp Ther Med 7: 1388-1392, 2014.

29. Oltvai ZN, Milliman CL and Korsmeyer SJ: Bcl-2 heterodimerizes in vivo with a conserved homolog, Bax, that accelerates programmed cell death. Cell 74: 609-619, 1993.

30. Bartling B, Holtz J and Darmer D: Contribution of myocyte apoptosis to myocardial infarction? Basic Res Cardiol 93: 71-84, 1998.

31. Jin S and Dai CL: Attenuation of reperfusion-induced hepatocyte apoptosis in associated with reversed bcl-2/bax ratio in hemi-hepatic artery-preserved portal occlusion. J Surg Res 174: 298-304, 2012. 